Research Paper

\title{
Identification of Hypermethylation in Hepatocyte Cell Adhesion Molecule Gene Promoter Region in Bladder Carcinoma
}

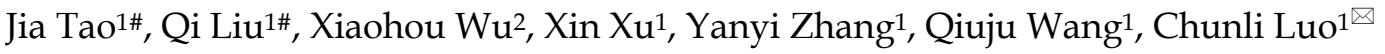 \\ 1. Department of Laboratory Diagnosis, Chongqing Medical University, Chongqing, 400016 China; \\ 2. Department of Urinary Surgery, First Hospital of Chongqing Medical University, Chongqing, 400016 China. \\ \# The authors contributed equally to this work and share first authorship.
}

$\triangle$ Corresponding author: Chunli Luo, Department of Laboratory Diagnosis, Chongqing Medical University, No.1, Yixueyuan Road, Yuzhong Restrict, Chongqing, 400016, China. E-mail: luochunli79@126.com. Fax: 023-68485005. Tel: 023-68485223.

( ) Ivyspring International Publisher. This is an open-access article distributed under the terms of the Creative Commons License (http://creativecommons.org/ licenses/by-nc-nd/3.0/). Reproduction is permitted for personal, noncommercial use, provided that the article is in whole, unmodified, and properly cited.

Received: 2013.04.12; Accepted: 2013.06.18; Published: 2013.II.II

\begin{abstract}
Background: Epigenetic regulation such as aberrant hypermethylation of $C_{p} G$ islands in promoter plays a key role in tumorigenesis. 5-Aza-2'-deoxycytidine (5-aza-CdR) which is a potent inhibitor of DNA methylation can reverse the abnormal hypermethylation of the silenced tumor suppressor genes (TSGs). It has been reported that hepatocyte cell adhesion molecule (hepaCAM) acts as a tumor suppressor gene and expression of its $\mathrm{mRNA}$ and protein were down-regulated in bladder cancer. Over-expression of hepaCAM can inhibit cancer growth and arrest renal cancer cells at G0/GI phase. In this study, we investigated the methylation status of hepaCAM gene, as well as the influence of 5-aza-CdR on expression of hepaCAM gene in bladder cancer cells. Methods: CpG islands in hepaCAM promoter and methprimers were predicted and designed using bioinformatics program. Methylation status of hepaCAM promoter was evaluated in bladder cancer tissues and two cell lines (T24 and BIU-87) by Methylation-specific PCR; Western blot and Immunofluorescence were used to detect expression of hepaCAM protein after 5-aza-CdR treatment; Flow cytometry assay was performed to determine effectiveness of 5 -aza-CdR on cell cycle profile. Results: CPG island in promoter of hepaCAM gene was hyper-methylated both in bladder carcinoma tissues and cell lines (T24 and BIU-87). Otherwise, aberrant methylation of its promoter was associated with its decreased expression. Hypermethylation of hepaCAM gene was reversed and expression of its mRNA and protein were re-activated in two cell lines by DNA methyltransferases inhibitor 5-aza-CdR. Flow cytometry assay demonstrated that 5-aza-CdR can inhibit growth of cancer cells by arresting cancer cells at GO/GI phase. Conclusion: Abnormal hypermethylation in CPG island of hepaCAM promoter is involved in absence of hepaCAM gene expression when bladder cancer occurs. Re-activation of hepaCAM gene by 5 -aza-CdR can inhibit growth of cancer cells and arrest cells at G0/GI phase.
\end{abstract}

Key words: hepaCAM; promoter; methylation; 5-aza-CdR; Bladder carcinoma.

\section{Introduction}

Hepatocyte cell adhesion molecule (hepaCAM) was discovered by Shali Shen in 2005[1]. It is a new type of cell adhesion molecules, belonging to the immunoglobulin superfamily. Earlier researchers found that the expression of hepaCAM in bladder cancer cells and tissues was decreased or absent, and over-expression of hepaCAM can inhibit tumor growth[2,3]. For its anti-cancer ability, hepaCAM has been presumed as a tumor suppressor gene. However, the mechanism of hepaCAM gene silencing in 
cancer is unknown.

DNA methylation is a covalent and reversible chemical modification, resulting from the addition of a methyl $(\mathrm{CH} 3)$ group at the carbon 5 position of the cytosine ring[4]. Aberrant DNA methylation plays an important role in regulating expression and biological function of TSGs(tumor suppressor genes), and contributing to tumorigenesis. Abundant previous studies showed that abnormal hypermethylation of $\mathrm{CpG}$ islands in TSGs promoter were devoted to genes silencing[5], such as RASSF1A, DAPK1, TFPI-2, p16 and CDH1[6-9]. 5-aza-CdR (5-Aza-2'-deoxycytidine) which is a DNA methyltransferases inhibitor has been proved to re-activate the expression of TSGs. For example, 5-aza-CdR induced re-expression of E-cadherin, p15 and p73 gene in cancers[10,11]. For its potent anticancer ability, 5-aza-CdR has been used for treatment of certain leukemia[12].

In this study, we have detected aberrant hypermethylation in promoter $\mathrm{CpG}$ islands of hepaCAM gene for the first time. Our results also indicated that hypermethylation of hepaCAM promoter significantly associated with decreased expression of its protein in bladder cancer. DNA methyltransferases inhibitor 5-aza-CdR can reverse its hypermethylation and lead to re-expression of hepaCAM gene.

\section{Materials and Methods}

\section{Cell lines and Patients}

T24 cell line was kindly donated by infectious diseases institute of Chongqing Medical University, China. BIU-87 cell line was purchased from cell bank of Wuhan University. They were cultured in RPMI1640 (Gibco, American) with 10\% fetal bovine serum (Gibco, American) at $37^{\circ} \mathrm{C}$ in a humidified atmosphere containing 5\% CO2 incubator. 30 cases of paired urothelial carcinoma samples and corresponding adjacent tissues were collected from the department of Urology at the First Affiliated Hospital of Chongqing Medical University, with patients' consent and institutional ethics committee. The tumor grades were evaluated according to the WHO standards (I: 5, II: 22, III: 3) and staging according to the $\mathrm{TNM}$ classification guidelines $(\mathrm{Ta} \sim \mathrm{T} 1,18 ; \mathrm{T} 2 \sim \mathrm{T} 4$, 12).

\section{Adenovirus Infection}

Cells were infected by advenovirus vetor and divided into the blank control group, the negative control group (infected with empty vetor $\mathrm{pAdH5}$ ) and the positive group (infected with pAdH5-hepaCAM).

\section{Promoter CpG islands prediction of hepaCAM}

Promoter prediction of hepaCAM gene was performed by FirstEF software[13]
(http://rulai.cshl.org/tools/FirstEF/). Then, CpG islands was predicted and methprimers for MSP was designed through the bioinformatics program[14] (http://www.urogene.org/cgi-bin/methprimer/met hprimer.cgi).

\section{Methylation-specific PCR}

Genomic DNA from cells and tissues was extracted according to the TIANamp Genomic DNA Kit's instruction (TIANGEN BIOTECH,China). The bisulfite modification procedure was carried out using EZ DNA Methylation-Gold ${ }^{\mathrm{TM}}$ Kit (Zymo Research). 500ng of genomic DNA was used in bisulfite treatment and the bisulfite-modified DNA was stored at $-80^{\circ} \mathrm{C}$. Amplification was started in a $20 \mathrm{ul}$ reaction volume including 10ul Hot start PCR mix (BIOTAKE, China), $1 \mathrm{ul}$ of each primer, $1 \mathrm{ul}$ of modified DNA and $7 \mathrm{ul}$ of $\mathrm{ddH}_{2} \mathrm{O}$. The cycling conditions were performed by initial denaturation at $95^{\circ} \mathrm{C}$ for $5 \mathrm{~min}$, followed by 40 cycles of $95^{\circ} \mathrm{C}$ for $50 \mathrm{~s}, 58.4^{\circ} \mathrm{C}(\mathrm{M}) / 61.8^{\circ} \mathrm{C}(\mathrm{U})$ for $50 \mathrm{~s}$, $72^{\circ} \mathrm{Cfor} 1 \mathrm{~min}$, and a final extension at $72^{\circ} \mathrm{C}$ for $10 \mathrm{~min}$. Primers for methylated DNA of hepaCAM were: Left M primer, 5'-AGAATTCGGTTTCGGAGTTTC-3'; Right M primer, 5'-CTAAACGACGACGAATATATC CG-3'. Primers for unmethylated DNA were: Left U primer, 5'-AGAATTTGGTTTTGGAGTTTTGA-3'; Right U primer, 5' - ACCCTAAACAACAACAAATA TATCCA-3'. The product sizes of the methylated and unmethylated amplification were $172 \mathrm{bp}$ and $175 \mathrm{bp}$, respectively. The products were visualized in a $3 \%$ polyacrylamide gel.

\section{Treatment with 5-aza-CdR}

5-aza-CdR was purchased from Sigma (USA). It dissolved in dimethylsulphoxide (DMSO) at a stock concentration of $3 \mathrm{mmol} / \mathrm{L}$ and stored at $-80^{\circ} \mathrm{C}$. The selection of drug concentrations was referring to previous MTT result[15]. Various concentrations of 5 -Aza-CdR (Table 1 ) were used for $72 \mathrm{~h}$ in the treatment. DMSO was used as a control.

Table I. The experiment grouping.

\begin{tabular}{lll}
\hline & T24 & BIU-87 \\
\hline 1 & DMSO & DMSO \\
2 & Blank & Blank \\
3 & $0.3 \mu \mathrm{mol} / \mathrm{L} 5$-aza-CdR & $0.1 \mu \mathrm{mol} / \mathrm{L} 5$-aza-CdR \\
4 & $1 \mu \mathrm{mol} / \mathrm{L} 5$-aza-CdR & $0.5 \mu \mathrm{mol} / \mathrm{L} 5$-aza-CdR \\
5 & $3 \mu \mathrm{mol} / \mathrm{L} 5$-aza-CdR & $1 \mu \mathrm{mol} / \mathrm{L} 5$-aza-CdR \\
6 & $10 \mu \mathrm{mol} / \mathrm{L} 5$-aza-CdR & $5 \mu \mathrm{mol} / \mathrm{L}$ 5-aza-CdR \\
\hline
\end{tabular}

\section{Western-blot for hepaCAM assay}

Total protein was extracted from bladder cancer cell and tissue using lysis buffer with 1mM Phenylmethanesulfonyl fluoride (PMSF). It was denatured in 
sodium dodecylbenzene sul fonate (SDS) loading buffer at $100^{\circ} \mathrm{C}$ for $5 \mathrm{~min}$. Protein was electrophoresed in $12 \%$ SDS-polyacrylamide gel and transfered to polyvinylidene membranes (PVDF) (Milliproe). The membranes were blocked with 5\% skim milk in TBST and then were immunoblotted overnight at $4^{\circ} \mathrm{C}$ using anti-hepaCAM antibody (Proteintech, American), $\beta$-actin antibody (Santa Cruz,CA). The membranes were incubated with a peroxidase-conjugated goat anti-rabbit or mouse sencondary antibody for $1 \mathrm{~h}$ at room temperature after being washed by TBST. Proteins were detected using a chemiluminescence (ECL) reagent (Milliproe) by western blotting detection and analysis system.

\section{Immunofluorescence analysis}

Cells cultured on coverslips were treated with 5 -aza-CdR, and then fixed with $4 \%$ paraformaldehyde for 10 minutes. They were blocked in $5 \%$ goat serum for $1-2 \mathrm{~h}$ at room temperature before permeabilized with $0.1 \%$ Triton X-100 for 15 minutes. Anti-hepaCAM antibody was incubated overnight at $4{ }^{\circ} \mathrm{C}$ followed by incubation of FITC-labeled secondary antibody for $1 \mathrm{~h}$ at room temperature in a moist container in dark. After washing with Phosphate Buffered Saline (PBS), slides were nuclear stained with 4,6-diamino-2-phenyl indole (DAPI). Fluorescence was visualized by fluorescence microscopy.

\section{Flow cytometry assay}

After treatment with 5-aza-CdR and infection with adenovirus, cells were harvested and washed with PBS three times. Cells fixed with $70 \%$ ethanol were centrifuged at $1000 \times \mathrm{g}$ for $10 \mathrm{~min}$ to remove ethanol, resuspended in staining solution $(1 \times \mathrm{PBS}$, $0.1 \%$ Triton $\mathrm{X}-100,20 \mathrm{ug} / \mathrm{ml}$ propidium iodide and $100 \mathrm{ug} / \mathrm{ml}$ RNase A) and incubated for 30min at room temperature. Cells were subjected to cell cycle analysis by FACS.

\section{Statistical analysis}

All the statistical analysis was performed with SPSS 16.0 software using One-way ANOVA, Paired-Samples $\mathrm{T}$ test and Spearman correlation analysis. $\quad P<0.05$ was considered statistically significant.

\section{Results}

\section{Effect of ectogenic hepaCAM gene on cell cy- cle profile}

Cell cycle profiles were analyzed by FCM (Fig.1). Overexpression of ectogenic hepaCAM in positive infected group arrested cells in G0/G1-phase $(p<0.05)$. There were no statistically significant difference between the pAdH5 cells and blank cells $(p>0.05)$ (Table 2).
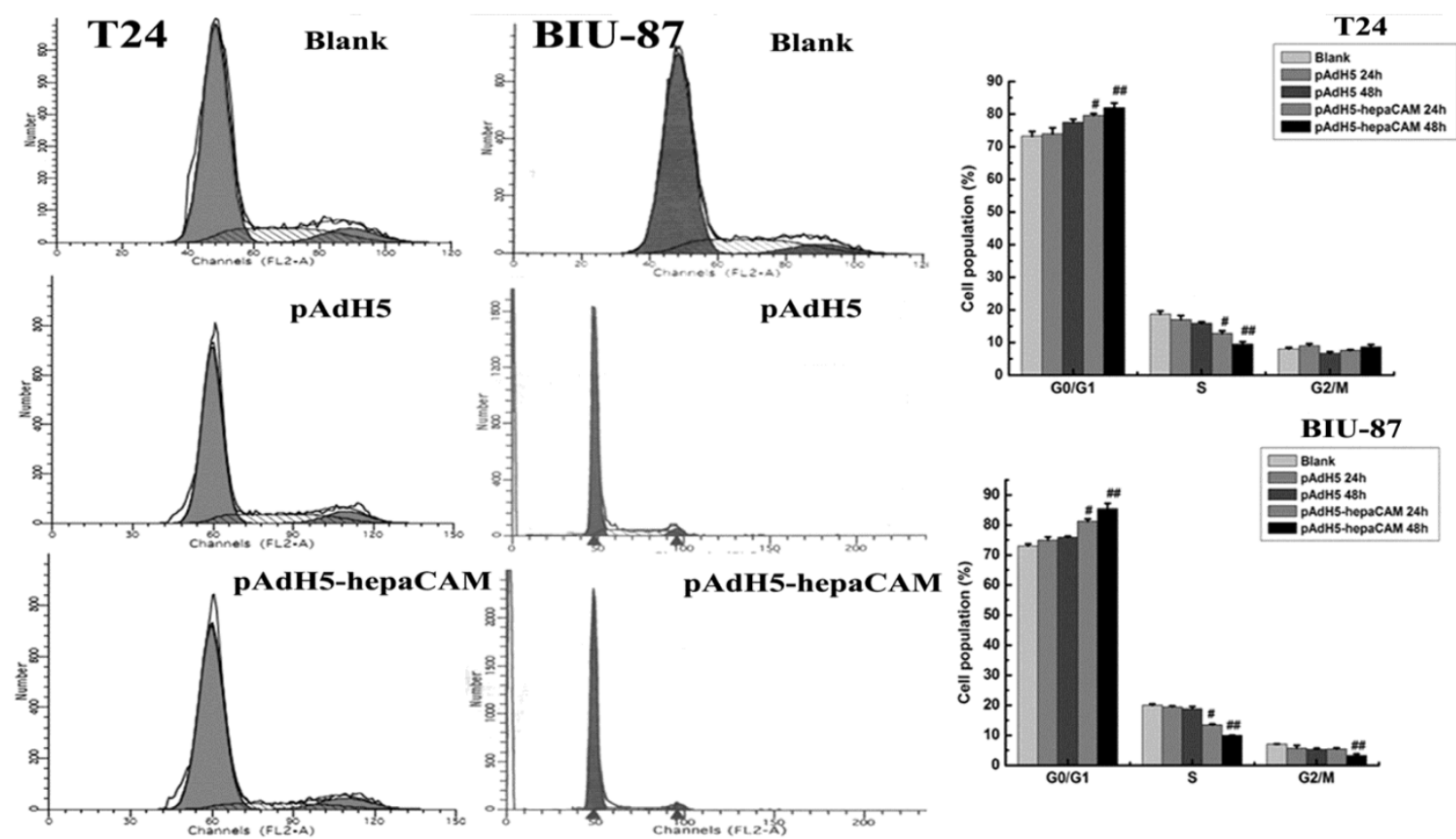

Fig I. Effect of ectogenic hepaCAM gene on cell cycle distribution. FCM measured the influence of pAdH5-hepaCAM on cell cycle distribution in two cell lines (T24 and BIU-87). Data are reported as mean $\pm S D(n=3)$. Cell percentage in $\mathrm{G} 0 / \mathrm{GI}$ phase was increased in $\mathrm{pAdH} 5$-hepaCAM group versus blank and pAdH5 group. The results revealed that hepaCAM can arrest cell cycle at G0/GI. \#: p<0.05, \#: p<0.0I. (Blank: cells without treatment; pAdH5: Adenovirus empty vector; pAdH5-hepaCAM: hepaCAM adenovirus vector). 

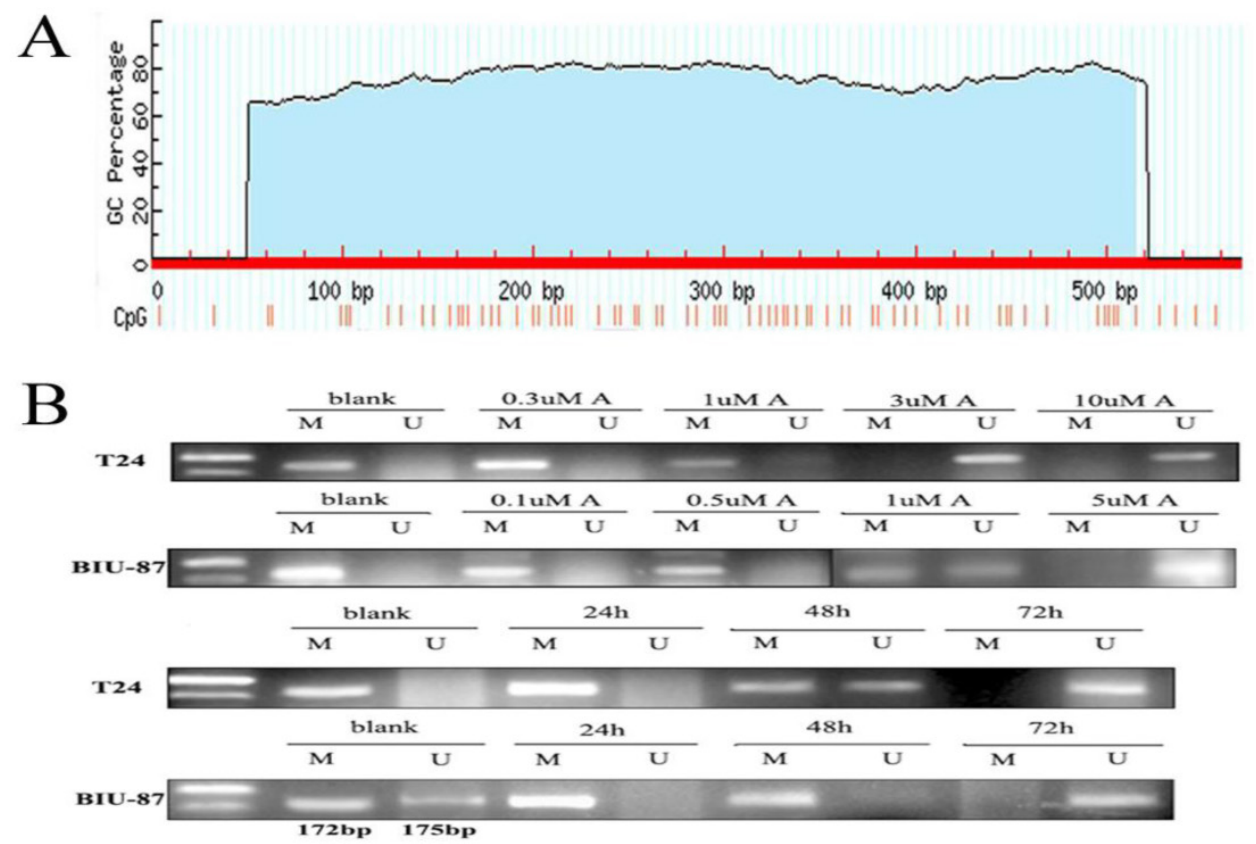

Fig 2. Prediction of CpG island in hepaCAM promoter and methylation status of hepaCAM promoter. (A) Promoter prediction of hepaCAM gene was performed by FirstEF software and the results showed that promoter of hepaCAM is from I 4857 to 15426 of gene sequence. CpG island of hepaCAM promoter was predicted using internet software, the blue region is $C_{p} G$ islands and the red thin lines are CpG sites; (B) MSP used to detect methylation status of hepaCAM gene in bladder cancer cell lines. In blank group, hepaCAM promoter was hypermethylated in T24 and BIU-87 cells, but treated with different concentration of 5-aza-CdR which can reverse the abnormal methylation. $3 \mathrm{uM}$ of 5 -aza-CdR can significantly repress the hypermethylation of hepaCAM gene in T24 cell line and $5 \mathrm{uM}$ for BIU-87 cell line. Meantime, different time of treatment also influence the effect of 5-aza-CdR. In two cells, the perfect time was 72h. (M: Methylation; U: Umethylation; A: 5-aza-CdR).

Table 2. Cell Cycle distribution of T24 and BIU-87 cell lines by FCM.

\begin{tabular}{llll}
\hline Groups & G0/G1 & S & G2/M \\
\hline blank & a) $73.27 \pm 1.45$ & $18.72 \pm 0.99$ & $8.01 \pm 0.49$ \\
& b) $72.98 \pm 0.64$ & $19.97 \pm 0.53$ & $7.04 \pm 0.14$ \\
pAdH5 & a) $73.97 \pm 1.78$ & $17.04 \pm 1.21$ & $8.98 \pm 0.62$ \\
& b) $74.93 \pm 1.03$ & $19.40 \pm 0.38$ & $5.67 \pm 0.94$ \\
pAdH5-hepaCAM & a) $81.93 \pm 1.48^{\#}$ & $9.57 \pm 0.66^{\#}$ & $8.62 \pm 0.78$ \\
& b) $85.40 \pm 1.80^{\#}$ & $10.03 \pm 0.07^{\#}$ & $3.28 \pm 0.54$ \\
\hline
\end{tabular}

a):T24 cell; b): BIU-87 cell; \#: $\mathrm{p}<0.05$.

\section{The promoter sequence of hepaCAM}

Prediction of hepaCAM promoter showed that promoter sequence is from 14857 to15426. There are $570 \mathrm{bp}$ in all. One $\mathrm{CpG}$ island was found in promoter sequence by Methprimer (Fig.2A). The blue region was $C p G$ islands and the red thin lines are $C p G$ sites.

\section{Methylation status of hepaCAM promoter in cells and demethylation of 5-aza-CdR}

Hypermethylation of hepaCAM promoter was apparently observed in both of T24 and BIU-87 cell lines. Besides, hepaCAM promoter was obviously demethylated by 3uM of 5-Aza-CdR in T24 cell line, and $5 \mathrm{uM}$ of 5 -Aza-CdR in BIU-87 cell line for $72 \mathrm{~h}$ (Fig.2B). Demethylation of hepaCAM promoter was not apparent in other concentrations. The expression of hepaCAM mRNA could be restored by 5 -Aza-CdR in T24 and BIU-87 cell lines that described before [15]. It was also detected by western-blot (Fig.3A) and Immunofluorescence (Fig.3B). The results revealed that hepaCAM protein was expressed after 5-Aza-CdR treatment. DNA methyltransferases inhibitor 5-Aza-CdR not only reverse the expression of hepaCAM mRNA but also its protein.

\section{Effect of 5-aza-CdR on cell cycle distribution}

According to previous researches, 5-Aza-CdR can re-activate the expression of TSGs, and has been used for treatment of certain leukemia. So the anti-tumor growth of 5-Aza-CdR in bladder cancer cells was analyzed using FCM. The results showed that cell cycle was arrested at G0/G1 after 5-aza-CdR treatment (Fig.4). Cells with 5-aza-CdR treatment were more statistically significant difference than blank cells and DMSO cells $(p<0.05)$ (Table 3$)$.

Table 3. Effect of 5 -aza-CdR on cell cycle distribution in T24 and BIU-87 cells detected by FCM.

\begin{tabular}{llll}
\hline Groups & G0/G1 & S & G2/M \\
\hline Blank & a) $71.79 \pm 0.44$ & $21.34 \pm 1.36$ & $6.87 \pm 1.53$ \\
& b) $65.10 \pm 0.55$ & $25.29 \pm 0.68$ & $9.61 \pm 0.52$ \\
5-aza-CdR/48h & a) $88.28 \pm 1.96^{*}$ & $5.85 \pm 0.22^{*}$ & $5.87 \pm 1.74$ \\
& b) $66.01 \pm 0.80$ & $25.69 \pm 1.74$ & $8.30 \pm 0.94$ \\
5 -aza-CdR/72h & a) $92.28 \pm 0.17^{\#}$ & $2.60 \pm 0.37^{\#}$ & $5.12 \pm 0.24$ \\
& b) $77.39 \pm 0.61^{\#}$ & $13.29 \pm 0.36^{\#}$ & $9.32 \pm 0.32$ \\
\hline
\end{tabular}

a):T24 cell; b): BIU-87 cell; \#: $p<0.01 ; *: p<0.05$. 
A

T24

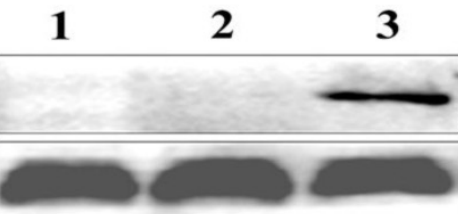

T24

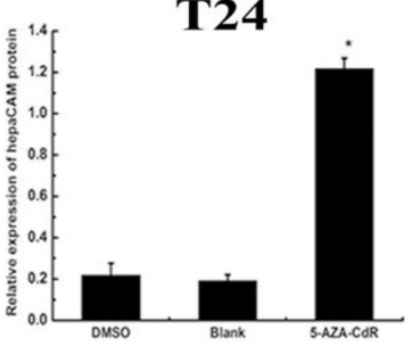

B

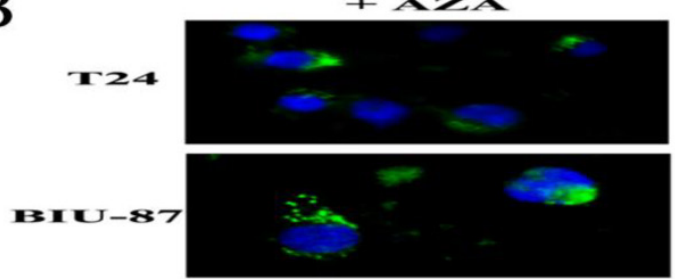

BIU-87

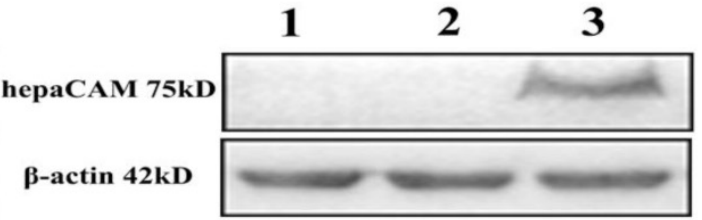

BIU-87

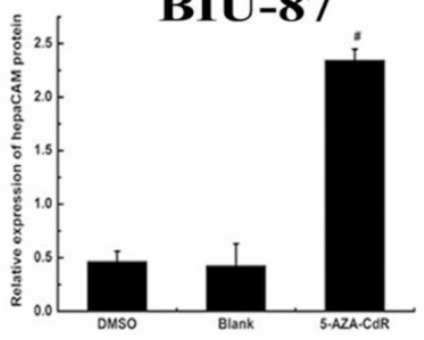

$-A>A$
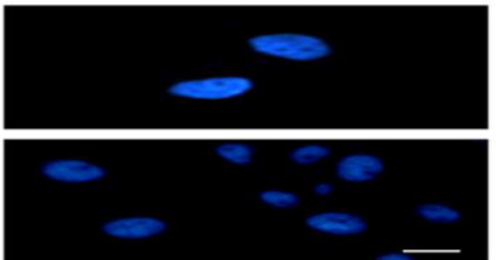

Fig 3. 5-aza-CdR reverse expression of hepaCAM protein. (A)After T24 and BIU-87 cells treated with 5-aza-CdR,total protein extracted from each group and I20ug of cell lysate was analyzed by Western-blot. The relative intensities of target bands were scanned by Quantity one software and normalized to $\beta$-actin levels. *: $p<0.01$, \#: $p<0.05$. The results showed that expression of hepaCAM protein was upregulated after 5-aza-CdR treatment in T24 and BIU-87 cell lines (I: DMSO; 2: Blank; 3: 5-aza-CdR.); (B) Immunofluorescence analyzed the re-expression of hepaCAM protein. Immunofluorescence demonstrated that hepaCAM protein re-expressed in T24 and BIU-87 cell lines with 5-aza-CdR treatment (I: cells with 5-aza-CdR treatment; 2: cells without 5-aza-CdR treatment). Image was obtained using Nikon Microscrope. Scale bar= 10 microns.
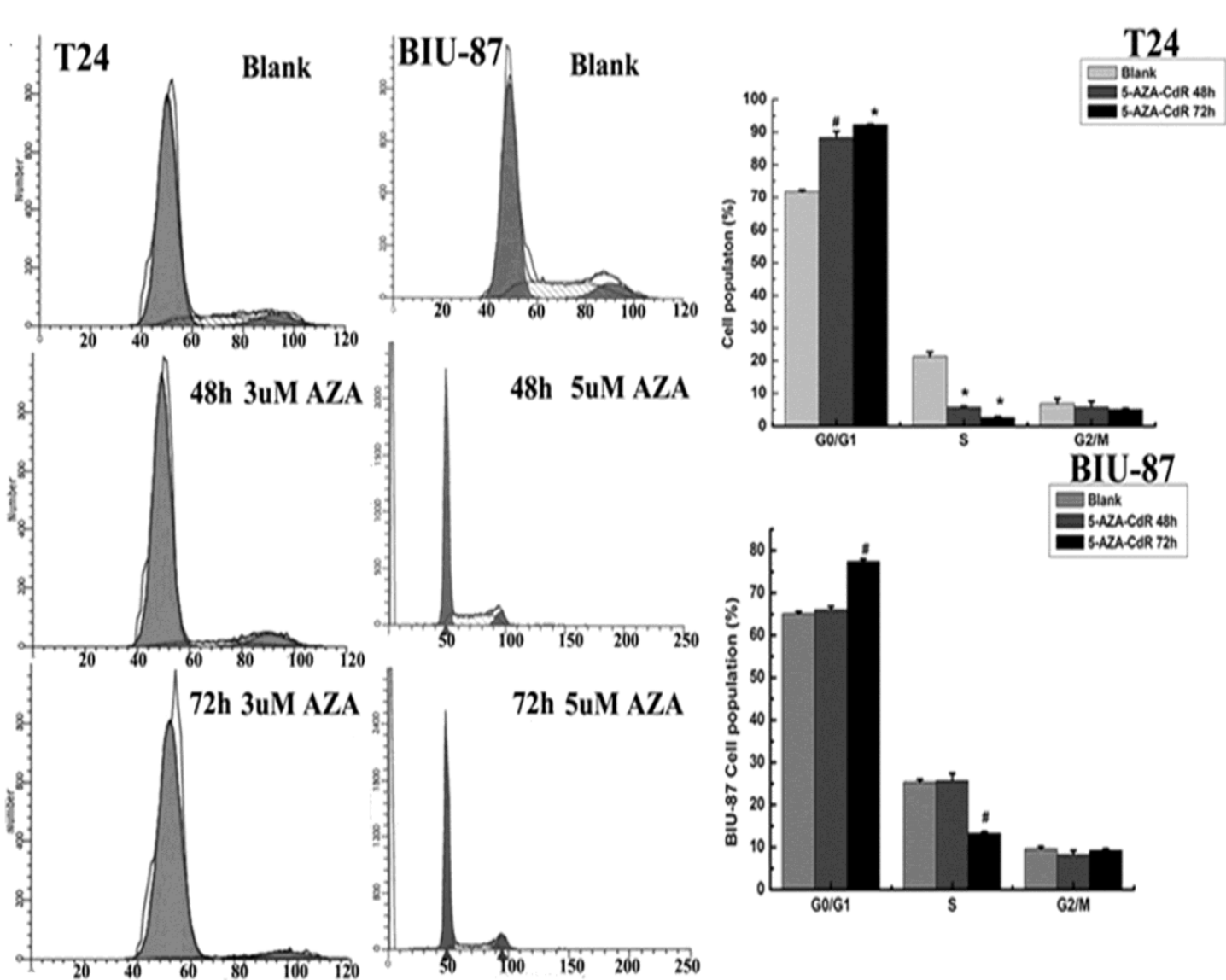

Fig 4. Effect of 5 -aza-CdR on cell cycle distribution. Cells treated with 5 -aza-CdR for different time and the cell percentage in each phase of three group were measured by flow cytometer. Data are reported as mean $\pm S D(n=3)$. The results showed that 5 -aza-CdR can arrest cell cycle at G0/GI. The effect of 5-aza-CdR on cell cycle distribution was much apparent at $72 \mathrm{~h}, \#: p<0.05, *: p<0.01$. 


\section{The methylation status of hepaCAM gene promoter and its expression of protein in paired tissues}

In vitro, hypermethylation of hepaCAM gene promoter was discovered in cell lines. The matter of wheather the same circumstance occurs in patients with bladder carcinoma is unknown. So methylation status of hepaCAM gene promoter was detected by MSP in bladder cancer tissues (Fig.5A). The positive rate of hepaCAM promoter methylation in tumor tissues was $83.33 \%$, which was obviously higher than that in adjacent normal bladder tissues (33.3\%, $p<0.05)$. Results of Western-blot revealed that hepaCAM protein mostly expressed in adjacent normal tissues of patients (Fig.5B). It expressed in only few tumor tissues $(p<0.01)$. Spearman correlation analysis demonstrated a negative correlation between expression of hepaCAM protein and its promoter hypermethylation (correlation coefficient $r=-0.894$, $p=0.000$ ) (Table 4).

Table 4. The correlation between expression of hepaCAM protein and hepaCAM promoter methylaion by MSP.

\begin{tabular}{ccc}
\hline & \multicolumn{2}{c}{ methylation } \\
\cline { 2 - 3 } parameters & + & - \\
\hline Deletion of hepaCAM protein & 24 & 0 \\
No change of hepaCAM protein & 1 & 5 \\
$\mathrm{r}$ & & -0.894 \\
$\mathrm{p}^{*}$ & & 0.000 \\
\hline
\end{tabular}

*: $p<0.01$ was considered statistically significant.

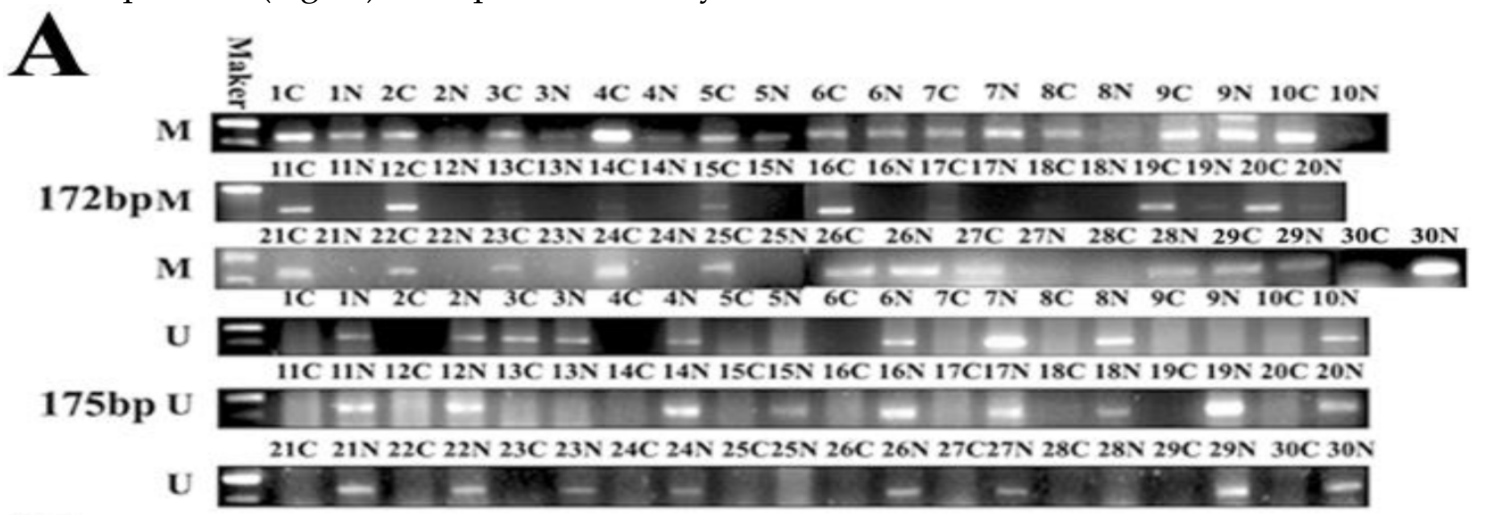

B

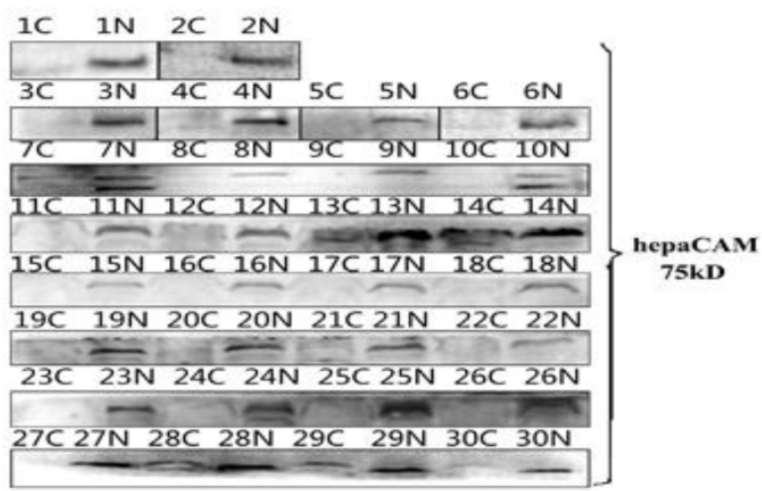

$\longrightarrow-\infty-\infty-\infty-a c t i n$

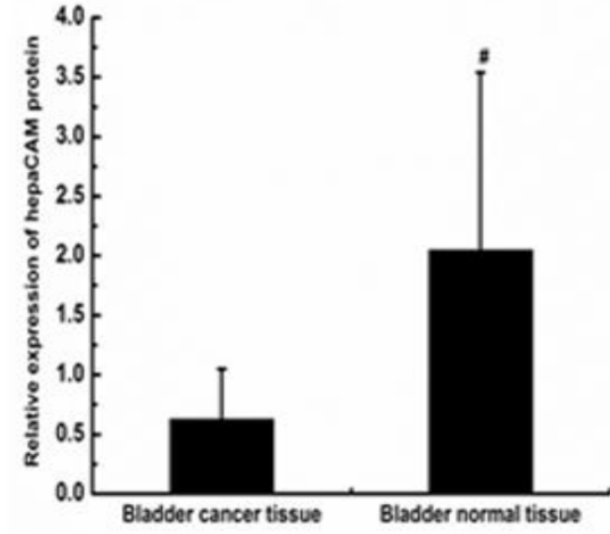

Fig 5. Methylation status of hepaCAM gene and expression of hepaCAM protein in bladder cancer tissue specimens. (A) Genome DNA were extracted and modified with treatment of sodium bisulfite. Modified DNA was used as template for MSP to detect methylation status of hepaCAM promoter in 30 paires patients. Results showed that 25 of 30 (83.3\%) patients' tumor tissues occurs hypermethylation of hepaCAM promoter, and 10 of 30 (33.3\%) patients' adjacent normal tissues occurs hypermethylation ( C: cancer tissue; N: normal tissue in side of cancer; $\mathrm{M}$ : methylation; $\mathrm{U}$ : unmethylation). (B)Total protein extracted from tissue specimens. Western-blot performed as described above to detect the expression of hepaCAM protein in 30 paires patients. In most of bladder cancer tissues, the expression of hepaCAM protein was lost, and it expressed in corresponding adjacent normal tissues. \#: $p<0.0 \mathrm{I}$ ( $\mathrm{C}$ : cancer tissue; $\mathrm{N}$ : normal tissue in adjacent cancer).

\section{Discussion}

Bladder cancer is the most common cancer in urologic neoplasms in China, and its incidence ranked 8th in systemic malignancy[16]. Bladder transitional cell carcinoma accounted for $90 \%$ of bladder tumor[17] and its biological behavior is complicated and changeable, with high heterogeneity and recurrent. The "gold standard" of current clinical diagnosis is cystoscopy with biopsy. This method is traumatic and difficult for patients with invisible tumors.

Methylation of DNA is a chemical modification process. The methyl group of S-adenosylmethionine transfers to the carbon five prime position of a cyto- 
sine pyrimidine ring of DNA under the action of DNA methyltransferase (DNMT), forming 5-methylcytosine. DNA methylation is an important mechanism for the inactivation of malignant tumor suppressor genes and occurs mainly in CpG islands[18]. Methylation of $\mathrm{CpG}$ islands in suppressor genes promoter occurs in most tumors. Moreover, abnormal DNA methylation occurred in early tumorigenesis, contributing as an ideal marker for early diagnosis of tumors[19]. Some studies have proved that epigenetic changes of multiple TSGs (tumor suppressor genes) were involved in breast pathogenesis[20]. Researchers also demonstrated that aberrant methylated genes played various functions in the cell cycle regulation, proliferation and adhesion[21]. Research of Jelilek J showed that aberrant DNA methylation is associated with CML progression[22]. In addition, Stephen JK' research suggested that abnormal methylation of CASP8, RASSF1, and NIS maybe early changed in thyroid tumorigenesis regardless of cell type[23]. These studies showed that methylation of TSGs promoter played a significant role in tumorigenesis.

As a tumor suppressor gene, the expression of hepaCAM was lost in human hepatocellular carcinoma (HCC)[24-26]. Previous studies also showed that hepaCAM played a similar role in transitional cell carcinoma of the bladder (TCCB) as well as in HCC[26]. To confirm that hypermethylation of hepaCAM promoter leads to its inactivation in bladder carcinoma, we analyzed the methylation status of hepaCAM promoter in bladder cancer cells and tumor tissues. We found a CpG island in hepaCAM promoter through Internet software (http://rulai.cshl.org/tools/FirstEF/ and http://ww w.urogene.org/methprimer/index1.html). Hypermethylation of hepaCAM promoter was detected by MSP in bladder cancer cells (T24 and BIU-87) and in $83.3 \%$ of patients. The expression of hepaCAM protein was lost in $80 \%$ patients. These results demonstrated that inactivation of hepaCAM gene may be caused by hypermethylation of promoter. Previous report[19]demonstrated that the methylation of hepaCAM gene was occurred at exon 2. Whether the methylation of hepaCAM gene promoter and exon 2 were the conjunct mechanism of gene silencing need in-depth investigate.

To further identify this, T24 and BIU-87 cell lines were treated with different concentration of 5 -aza-CdR to investigate the effect of 5 -aza-CdR on the expression of hepaCAM mRNA, protein and promoter methylation. 5-aza-CdR covalently binds DNA methyltransferase, which then become trapped and unable to catalyse the methylation of newly synthesized DNA strands[27]. Clinical trials found that
DNA methylation gradually disappears in the tumor cells from responders with 5-Aza-CdR treatment[27]. 5 -aza-CdR is phosphorylated by deoxycytidine kinase, and its end product, decitabine triphosphate, is incorporated into DNA[28]. Incorporation of a high concentration of decitabine triphosphate into DNA can inhibit DNA synthesis and induce cell cycle arrest[29-31]. 5-aza-CdR has been used in clinical phase III trials patients with Myelodysplastic syndrome (MDS)[28]. Ju Hee Kim's research showed that 5-Aza-CdR can reverse expression of CAMK2B and ARFGEF1, besides hypermethylation of CAMK2B and ARFGEF1can inhibit gene activity in cancer cells[32]. Trenton et al. showed that 5-aza-CdR significantly increased the expression of PHD3[33]. Previous studies demonstrated that treatment of 5-Aza-CdR in corresponding concentration for $72 \mathrm{~h}$ in T24 and BIU-87 cell lines can reverse the expression of hepaCAM mRNA[15]. These studies revealed that 5-Aza-CdR can also reverse the expression of hepaCAM protein. This drug affects the cell cycle distribution, arresting the cell cycle at G0/G1 phase. It also shows that 5 -aza-CdR can restrain bladder cancer cells' growth and reverse the hypermethylation of hepaCAM promoter.

In conclusion, hypermethylation occurs in hepaCAM promoter region. Demethylating drug (5-aza-CdR) can reverse its expression of mRNA and protein, and inhibit the growth of tumor cells. Hypermethylation of promoters is a major gene silencing mechanisms of hepaCAM in human bladder cancer. Further research is required to identify that which promoter sites mostly affects transcriptional level.

\section{Abbreviations}

hepaCAM, hepatocyte cell adhesion molecule; 5-aza-CdR, 5-Aza-2'-deoxycytidine; TGSs, tumor suppressor genes; MSP, Methylation-specific polymerase chain reaction; FCM, Flow Cytometry

\section{Acknowledgements}

This work was supported by National Natural Science Foundation of P.R. China (Grant No. 81072086).

\section{Competing Interests}

The authors have declared that no competing interest exists.

\section{References}

1. Chung Moh M, Hoon Lee L, Shen S, et al. Cloning and characterization of hepaCAM, a novel Ig-like cell adhesion molecule suppressed in human hepatocellular carcinoma. J Hepatol. 2005;42(6): 833-41.

2. He $Y, W u ~ X, ~ L u o ~ C$, et al. Functional significance of the hepaCAM gene in bladder cancer. BMC Cancer. 2010 Mar 8;10:83. 
3. Yang $\mathrm{S}, \mathrm{Wu} \mathrm{X}$, Luo $\mathrm{C}$, et al. Expression and clinical significance of hepaCAM and VEGF in urothelial carcinoma. World J Urol. 2010;28(4):473-8.

4. Partha M. Das, Rakesh Singal.DNA Methylation and Cancer. J Clin Oncol, 2004;22:4632-4642

5. Adrian Bird. DNA methylation patterns and epigenetic memory. GENES \& DEVELOPMENT, 2012;16:6-21.

6. Ahmed IA, Pusch CM, Hamed T, et al. Epigenetic alterations by methylation of RASSF1A and DAPK1 promoter sequences in mammary carcinoma detected in extracellular tumor DNA. Cancer Genet Cytogenet. 2010;199(2):96-100

7. Wang S, Xiao X, Zhou X, et al. TFPI-2 is a putative tumor suppressor gene frequently inactivated by promoter hypermethylation in nasopharyngeal carcinoma. BMC Cancer, 2010; 9:10-617.

8. Barzily-Rokni M, Friedman N, Ron-Bigger S, et al. Synergism between DNA methylation and macroH2A1 occupancy in epigenetic silencing of the tumor suppressor gene p16(CDKN2A). Nucleic Acids Res. 2011; 39(4):1326-35.

9. Caldeira JR, Prando EC, Quevedo FC, et al. CDH1 promoter hypermethylation and E-cadherin protein expression in infiltrating breast cancer. BMC Cancer, 2006; 6:48.

10. Jeong-Seok Nam, Yoshinori Ino, Yae Kanai, et al. 5-Aza-2-deoxycytidine restores the E-cadherin system in E-cadherin-silenced cancer cells and reduces cancer metastasis. Clinical \& Experimental Metastasis, 2004; 21: 49-56.

11. Nuno J. Farinha, Sepideh Shaker, Maryse Lemaire, et al. Momparler. Activation of Expression of p15, p73 and E-Cadherin in Leukemic Cells by Different Concentrations of 5-Aza-2'-Deoxycytidine (Decitabine). ANTICANCER RESEARCH, 2004;24: 75-78.

12. Pinto, A. \& Zagonel, V. Leukemia 1993; 7:51-60.

13. Ramana V. Davuluri, Ivo Grosse, Michael Q. Zhang. Computational identification of promoters and first exons in the human genome. Nature Genetics, 2001; 29:412-417.

14. Li LC, Dahiya R. MethPrimer: designing primers for methylation PCRs. Bioinformatics, 2002; 18(11):1427-31.

15. Pan $\mathrm{C}, \mathrm{Wu} \mathrm{X}$, Luo $\mathrm{C}$, et al. Exon 2 methylation inhibits hepaCAM expression in transitional cell carcinoma of the bladder. Urol Int. 2010; 85(3):347-54.

16. Babjuk MO, Osterlinck W, Sylvester R, et al. EAU guidelines on-nonmuscle -carcinoma of the bladder. EAU guidelines, 2009, 33(4):361-371

17. Oosterlinck W, Lobel B, Jakse G, et al. Guidelines on bladder cancer. Eur Urol, 2002;41: 105-112.

18. Jones PA, Baylin SB. The fundamental role of epigenetic events in cancer. Nat Rev Genet, 2002;3:415-428.

19. Esteller, M, Corn, PG. Agene hypermethylation profile of human cancer. Cancer research, 2001;61(8):3225-29.

20. Xiang TX, Yuan Y, Li LL, et al. Aberrant promoter CpG methylation and its translational applications in breast cancer. Chin J Cancer, 2011; doi: 10.5732/cjc.011.10344.

21. Marzese DM, Gago FE, Orozco JI, et al. Aberrant DNA methylation of cancer -related genes in giant breast fibroadenoma:a case report[J]. J Med Case Reports, 2011;5(1):516.

22. Jelinek J, Gharibyan V, Estecio MR et al. Aberrant DNA methylation is associated with disease progression, resistance to imatinib andshortened survival in chronic myelogenous leukemia. PLoS One, 2011; 6(7):e22110.

23. Stephen JK, Chitale D, Narra V, et al. DNA methylation in thyroid tumorigenesis. Cancers (Basel). 2011; 3(2):1732-1743.

24. Moh MC, Zhang $\mathrm{C}$, Luo $\mathrm{C}$, et al. Structural and functional analyses of a novel ig-like cell adhesion molecule, hepaCAM, in the human breast carcinoma MCF7 cells. J Biol Chem, 2005;280(29):27366-27374.

25. Lee LH, Moh MC, Zhang T, et al. The immunoglobulin-like cell adhesion molecule hepaCAM induces differentiation of human glioblastoma U373-MG cells. J Cell Biochem 2009;15:1129-1138.

26. Moh MC, Zhang T, Lee LH, et al. Expression of hepaCAM is downregulated in cancers and induces senescence-like growth arrest via a p53/p21-dependent pathway in human breast cancer cells. Carcinogenesis 2008; 29(12):2298-2305.

27. Grønbaek K, Treppendahl M, Asmar F, et al. Epigenetic changes in cancer as potential targets for prophylaxis and maintenance therapy. Basic Clin Pharmacol Toxicol. 2008;03(5):389-96.

28. Oki Y, Issa JP. Recent Clinical Trials in Epigenetic Therapy. Rev Recent Clin Trials. 2006; 1(2):169-82.

29. Li LH, Olin EJ, Fraser TJ, et al. Phase specificity of 5-azacytidine against mammalian cells in tissue culture. Cancer Res, 1970;30:2770-5.

30. Cihak A, Vesely J. Prolongation of the lag period preceding the enhancement of thymidine and thymidylate kinase activity in regenerating rat liver by 5-azacytidine. Biochem Pharmacol, 1972;21:3257-65.

31. Cihak A, Vesely J, Skoda J. Azapyrimidine nucleosides: metabolism and inhibitory mechanisms. Adv Enzyme Regul, 1985;24:335-54.

32. Kim JH, Kim TW, Kim SJ. Downregulation of ARFGEF1 and CAMK2B by promoter hypermethylation in breast cancer cells. BMB Rep. 2011; 44(8):523-8.

33. Place TL, Fitzgerald MP, Venkataraman S, et al. Aberrant Promoter CpG Methylation Is a Mechanism for Impaired PHD3 Expression in a Diverse Set of Malignan Cells. PLoS One. 2011; 6(1):e14617. 